\title{
Assessing the need for resuscitative endovascular balloon occlusion of the aorta (REBOA) for management of non-compressible hemorrhage at a Canadian urban trauma centre: a retrospective chart review.
}

Elizabeth Purssell ( $\square$ elizabeth.purssell@gmail.com )

Royal Columbian Hospital https://orcid.org/0000-0001-5050-8660

\section{Sean Patrick}

The University of British Columbia, The University of British Columbia

Joseph Haegert

Royal Columbian Hospital, The University of British Columbia

\section{Vesna Ivkov}

Fraser Health Authority: Fraser Health, The University of British Columbia John Taylor

Royal Columbian Hospital, The University of British Columbia

Original research

Keywords: Resuscitative Endovascular Balloon Occlusions of the Aorta (REBOA), Trauma, Resuscitation. Hemorrhage, Canada

Posted Date: September 8th, 2021

DOl: https://doi.org/10.21203/rs.3.rs-836732/v1

License: (c) (i) This work is licensed under a Creative Commons Attribution 4.0 International License. Read Full License 


\section{Abstract \\ Background}

Resuscitative endovascular balloon occlusion of the aorta (REBOA) is a less invasive alternative to resuscitative thoracotomy (RT) for life threatening, infra-diaphragmatic, non-compressible hemorrhage from trauma. Existing evidence surrounding the efficacy of REBOA is conflicting; nevertheless, expert consensus suggests that REBOA should be considered in select trauma patients. There has been a paucity of studies that evaluate the potential utility of REBOA in the Canadian setting. The study objective was to evaluate the percentage of trauma patients presenting to a Level 1 Canadian trauma centre that would have met criteria for REBOA.

\section{Methods}

We conducted a retrospective chart review of patients recorded in the British Columbia Trauma Registry who warranted a trauma team activation (TTA) at our institution. We identified REBOA candidates using pre-defined criteria based on published guidelines. Each TTA case was screened by a reviewer, and then each Potential Candidate was reviewed by a panel of trauma physicians for determination of final candidacy.

\section{Results}

Fourteen patients were classified as Likely REBOA Candidates (2.2\% of TTAs, median age 46.1 years, $64.3 \%$ female). These patients had a median Injury Severity Score of 31.5 (IQR 26.8). The main sources of hemorrhage in these patients were from abdominal injuries $(71.4 \%)$ and pelvic fractures $(42.9 \%)$.

\section{Conclusion}

The percentage of patients who met criteria for REBOA is similar to that of RTs performed at our Canadian institution. While REBOA would be performed infrequently, it is a less-invasive alternative to RT, which could be a potentially life-saving procedure in a small group of the most severely injured trauma patients.

\section{Introduction}

Massive exsanguination resulting in circulatory collapse is one of the leading causes of preventable death in trauma. ${ }^{1-7}$ The cornerstone of the emergency management of massive hemorrhage is obtaining control of the bleeding. However, source control becomes a challenge when the source of major hemorrhage involves non-compressible regions such as the trunk and ilio-junctional regions. ${ }^{8,9}$ Consequently, noncompressible hemorrhage represents the majority of traumatic exsanguination fatalities accounting for $45-60 \%$ of deaths. ${ }^{10,11}$ Historically, the only means of attempting to control this type of bleeding was to 
perform a resuscitative thoracotomy (RT) as a bridge to either definitive surgical management in the operating room (OR) or arterial embolization by interventional radiology (IR) ${ }^{8,9}$ This highly invasive procedure is associated with high levels of mortality and complications. ${ }^{12}$

Within the last 10 years, innovation has led to the development and popularization of a second option for the management of life threatening non-compressible hemorrhage. Resuscitative endovascular balloon occlusion of the aorta (REBOA) involves inflation of a balloon in the aorta as a means of reducing blood flow to the distal hemorrhage site as well as preferentially directing blood flow to critical organs including the heart and brain. 8,9,13-19 Although REBOA is less invasive than RT, the existing evidence surrounding the efficacy of REBOA is conflicted; ${ }^{16,20-27}$ nevertheless, expert consensus suggests that REBOA should be considered in select severely injured patients. ${ }^{28,29}$

Many US centres that see high volumes of trauma and perform regular RTs have already adopted REBOA at their institutions. Globally, REBOA is available in many major centres, including in the UK, Europe, and Japan $^{23,24,27,30,31}$. However, the pattern and volume of trauma is different in Canada. ${ }^{32-34}$ Although some Canadian institutions are currently performing REBOA, there has been a paucity of studies evaluating the potential utility of this tool in the Canadian context ${ }^{35}$, and it has yet to be adopted as standard of care in Canada. A recent editorial has highlighted the need for further research in the Canadian setting prior to widespread adoption of REBOA ${ }^{36}$. Our study is a Canadian-based trauma population-driven assessment for the need for acquisition of REBOA at a tertiary care, Level 1 trauma centre. The objective of this study was to evaluate the percentage of trauma patients at our Canadian institution that sustained injury resulting in life-threatening hemorrhage below the diaphragm where REBOA would have been indicated as a component of the emergency department (ED) resuscitation.

\section{Methods}

\section{Study Design, Time Period, and Setting}

This retrospective descriptive study was conducted at the Royal Columbian Hospital, which is a Level 1 Canadian trauma centre that sees approximately 1000 trauma consults annually. We used medical record data for trauma patients captured in the British Columbia Trauma Registry (BCTR) who presented to our ED between January 1, 2016 and December 31, 2018. The BCTR is a comprehensive and organized provincial trauma surveillance and data collection system that is regularly quality checked.

\section{Population}

All BCTR cases that were trauma team activations (TTAs) were screened for inclusion in this study. TTAs are called for patients when there is concern for severe injury based on a set of criteria established by the Health Authority (Appendix A).

\section{Inclusion and Exclusion Criteria}


The inclusion and exclusion criteria for REBOA candidacy were chosen based on suggested REBOA protocols published in the literature ${ }^{37-40}$ and the indications published in the 2018 and 2019 American College of Surgeons and American College of Emergency Physicians guidelines. ${ }^{28,29}$

Cases must have had one of the following inclusion criteria to be considered a REBOA candidate:

1. Patients that sustained blunt trauma with a suspected infra-diaphragmatic injury that presented to the ED with a pulse and a systolic blood pressure less than $90 \mathrm{mmHg}$ that were transient or nonresponders to resuscitation

2. Patients that sustained penetrating trauma to the torso below the diaphragm that presented to the ED with a pulse and a systolic blood pressure less than $90 \mathrm{mmHg}$ that were transient or non-responders to resuscitation

3. Patients that sustained blunt trauma with a suspected infra-diaphragmatic injury that presented pulseless to the ED but had an organized rhythm on ECG or organized cardiac contraction seen on point-of-care ultrasound

4. Patients that sustained penetrating trauma to the torso below the diaphragm that presented pulseless to the ED and had less than 15 minutes of CPR.

Transient response to resuscitation was defined as a blood pressure that increased above $90 \mathrm{mmHg}$ with two units of blood product resuscitation for less than 15 minutes. Non-response to resuscitation was defined as a blood pressure that failed to increase above $90 \mathrm{mmHg}$ with two units of blood product resuscitation or patients that required inotropic support.

The infra-diaphragmatic torso included the abdomen, pelvis, and ilio-junctional areas. Suspected infradiaphragmatic injury in blunt trauma patients was defined as a patient with a:

1. Positive computed tomography (CT) scan for intra-abdominal or pelvic hemorrhage, or

2. Pelvic fracture on x-ray, or

3. Positive point-of-care abdominal ultrasound for free fluid, or

4. Clear clinical sign of abdominal, pelvic, or ilio-junctional hemorrhage such as open hemorrhage.

Cases must not have had any of the following exclusion criteria to be considered a REBOA candidate:

1. Non-traumatic cause of hemorrhage or cardiac arrest including myocardial infarction, toxicological arrest, hypoxia, or any other cause of arrest not related to the traumatic incident

2. Patients that sustained blunt trauma and presented pulseless to the ED but had no organized rhythm on the ECG or organized cardiac contractility on point-of-care ultrasound

3. Patients that sustained penetrating trauma but had been pulseless for greater than 15 minutes

4. Trauma patients that also had evidence of a major thoracic injury including a cardiac injury, a supradiaphragmatic aortic injury, or other major thoracic vascular injury

5. Trauma patients in which their main source of hemorrhage was from an extremity that was controllable with tourniquet or direct pressure 
6. Trauma patients that also sustained a devastating head injury

7. Trauma patients that also had a major neck injury.

\section{Outcome Measures}

The primary outcome was identification of REBOA candidacy according to inclusion and exclusion criteria. Additionally, patient characteristics, clinical variables, and details on the traumatic event were collected for each case. These included patient sex and age, comorbidities, mechanism of injury, injury severity score (ISS), and presenting vitals. Specific management variables were also collected: blood products transfused within 1, 2, 4, and 24-hours after initiation of resuscitation; activation of massive transfusion protocol; tourniquet placement; pelvic binder placement; and use of resuscitative thoracotomy and aortic cross-clamping. The details collected contribute to the clinical decision to use REBOA during ED resuscitation and characterize the types of patients that are REBOA candidates. The main source of hemorrhage identified was categorized as pelvic fractures, abdominal solid organ injury (liver, spleen, kidney, pancreas), lower extremity amputation, or major vascular injury.

\section{Data Collection}

Records for each TTA were accessed through the Health Authority's Patient Care Information System (PCIS). Each case was screened by a reviewer for inclusion and exclusion criteria and data from each identified case was extracted using a standardized data collection form (Appendix B). The data was subsequently copied into a secure electronic database.

Based on the defined indications for REBOA, the reviewers classified each case as whether or not the patient would have been a Potential or Likely REBOA Candidate. Potential Candidates were defined as those that met inclusion criteria without any exclusion criteria. Likely Candidates were those that met criteria for REBOA candidacy and received four or more units of packed red blood cells. All cases that were identified as a Likely REBOA Candidate were then subsequently reviewed by a panel of trauma physicians for final determination of candidacy. Additionally, the two reviewers assessed a sample of 60 cases ( $9.4 \%$ of charts) to ensure inter-rater reliability of case analysis.

\section{Statistical Analysis}

Descriptive statistics were utilized. Parametric continuous data was expressed using means and standard deviation (STD), non-parametric continuous data was expressed using median and interquartile ranges (IQR), and categorical data was expressed using $n$ values (\%). The Kappa statistic was used to express inter-rater agreement between chart reviewers.

\section{Results}

The BCTR recorded 1237 consults to the trauma service at our centre from January 1, 2016 to December 31, 2018. In total, 635 of these consults were TTAs. Following review, 21 patients were classified as Potential REBOA Candidates (3.3\% of TTAs) and 14 patients were classified as Likely REBOA Candidates 
(2.2\% of TTAs; $1.1 \%$ of total trauma consults) (Fig. 1 ). Table 1 provides descriptions of each case that met candidacy criteria.

Table 1

Case descriptions of Likely REBOA Candidates

\begin{tabular}{|ll|}
\hline BCTR & British Columbia Trauma Registry \\
\hline CPR & Cardiopulmonary resuscitation \\
\hline ED & Emergency department \\
\hline EHS & Emergency Health Services \\
\hline HR & Heart rate \\
\hline IQR & Interquartile range \\
\hline IR & Interventional radiology \\
\hline OR & Operating room \\
\hline REBOA & Resuscitative endovascular balloon occlusion of the aorta \\
\hline RT & Resuscitative thoracotomy \\
\hline SBP & Systolic blood pressure \\
\hline STD & Standard deviation \\
\hline TTA & Trauma team activation \\
\hline USA & United States of America \\
\hline
\end{tabular}

The inclusion criteria met by the 14 Likely Candidates were as follows: ten were blunt trauma with a pulse and SBP $<90 \mathrm{mmHg}(71.4 \%)$, three were penetrating trauma with a pulse and SBP $<90 \mathrm{mmHg}(21.4 \%)$, and one was a blunt trauma and pulseless (7.2\%). No patients met the penetrating trauma and pulseless criteria in our cohort. The mechanisms of injury sustained by Likely Candidates are reported in Table 2. 
Table 2

Characteristics of Potential and Likely REBOA Candidate injuries

\begin{tabular}{|c|c|c|c|c|c|c|}
\hline $\begin{array}{l}\text { Candidate } \\
\#\end{array}$ & $\begin{array}{l}\text { Likely } \\
\text { Candidate }\end{array}$ & $\begin{array}{l}\text { Inclusion } \\
\text { Criteria Met }\end{array}$ & $\begin{array}{l}\text { Primary } \\
\text { Source of } \\
\text { Hemorrhage }\end{array}$ & $\begin{array}{l}\text { Immediate } \\
\text { Surgical } \\
\text { Intervention }\end{array}$ & $\begin{array}{l}\text { Death in } \\
\text { Hospital }\end{array}$ & $\begin{array}{l}\text { Case } \\
\text { Description }\end{array}$ \\
\hline 1 & Yes & $\begin{array}{l}\text { Blunt, with } \\
\text { pulse }\end{array}$ & $\begin{array}{l}\text { Abdominal } \\
\text { solid organ } \\
\text { injury }\end{array}$ & $\begin{array}{l}\text { IR } \\
\text { OR }\end{array}$ & No & $\begin{array}{l}\text { Elderly patient } \\
\text { with fall from } \\
\text { standing. } \\
\text { Presented } 4 \\
\text { days after fall. } \\
\text { Spleen and } \\
\text { liver } \\
\text { lacerations. } \\
\text { Received } 38 \\
\text { units of blood } \\
\text { product in } 24 \\
\text { hours. Femoral } \\
\text { catheterization } \\
\text { may not have } \\
\text { been } \\
\text { successful due } \\
\text { to } \\
\text { atherosclerosis } \\
\text { noted. }\end{array}$ \\
\hline 2 & No & $\begin{array}{l}\text { Blunt, with } \\
\text { pulse }\end{array}$ & $\begin{array}{l}\text { Abdominal } \\
\text { solid organ } \\
\text { injury }\end{array}$ & None & No & $\begin{array}{l}\text { Dirt bike crash. } \\
\text { Required } 3 \\
\text { units pRBC. } \\
\text { Stabilized } \\
\text { after. }\end{array}$ \\
\hline 3 & Yes & $\begin{array}{l}\text { Penetrating, } \\
\text { with pulse }\end{array}$ & $\begin{array}{l}\text { Abdominal } \\
\text { solid organ } \\
\text { injury }\end{array}$ & OR & No & $\begin{array}{l}\text { Gunshot } \\
\text { wound to } \\
\text { abdomen. } \\
\text { Liver } \\
\text { laceration. } \\
\text { Received } 50 \\
\text { units of blood } \\
\text { product in } 24 \\
\text { hours. }\end{array}$ \\
\hline 4 & Yes & $\begin{array}{l}\text { Blunt, with } \\
\text { pulse }\end{array}$ & Pelvic fracture & IR & No & $\begin{array}{l}\text { Pedestrian } \\
\text { struck by a } \\
\text { vehicle. } \\
\text { Received } 17 \\
\text { units of blood } \\
\text { product in } 24 \\
\text { hours. IR } \\
\text { embolization } \\
\text { successful. }\end{array}$ \\
\hline 5 & Yes & $\begin{array}{l}\text { Blunt, with } \\
\text { pulse }\end{array}$ & Pelvic fracture & None & Yes & $\begin{array}{l}\text { Pedestrian } \\
\text { struck by a } \\
\text { vehicle. } \\
\text { Arrested in the } \\
\text { CT scanner } \\
\text { and died. }\end{array}$ \\
\hline
\end{tabular}




\begin{tabular}{|c|c|c|c|c|c|c|}
\hline $\begin{array}{l}\text { Candidate } \\
\#\end{array}$ & $\begin{array}{l}\text { Likely } \\
\text { Candidate }\end{array}$ & $\begin{array}{l}\text { Inclusion } \\
\text { Criteria Met }\end{array}$ & $\begin{array}{l}\text { Primary } \\
\text { Source of } \\
\text { Hemorrhage }\end{array}$ & $\begin{array}{l}\text { Immediate } \\
\text { Surgical } \\
\text { Intervention }\end{array}$ & $\begin{array}{l}\text { Death in } \\
\text { Hospital }\end{array}$ & $\begin{array}{l}\text { Case } \\
\text { Description }\end{array}$ \\
\hline 6 & No & $\begin{array}{l}\text { Blunt, with } \\
\text { pulse }\end{array}$ & $\begin{array}{l}\text { Other - crush } \\
\text { injury }\end{array}$ & None & Yes & $\begin{array}{l}\text { Trapped in a } \\
\text { truck for } 3 \\
\text { days prior to } \\
\text { ED } \\
\text { presentation. } \\
\text { Died of } \\
\text { acidosis } \\
\text { secondary to } \\
\text { crush injury to } \\
\text { bilateral legs. }\end{array}$ \\
\hline 7 & Yes & $\begin{array}{l}\text { Blunt, with } \\
\text { pulse }\end{array}$ & Pelvic fracture & None & No & $\begin{array}{l}\text { Run over and } \\
\text { trapped } \\
\text { beneath a car. } \\
\text { Sustained } \\
\text { pelvic and } \\
\text { femur } \\
\text { fractures. } \\
\text { Received } 11 \\
\text { units of blood } \\
\text { product in } 24 \\
\text { hours. Did not } \\
\text { require urgent } \\
\text { OR but REBOA } \\
\text { may have been } \\
\text { deployed in the } \\
\text { setting of a } \\
\text { pelvic fracture } \\
\text { prior to } \\
\text { stabilization. }\end{array}$ \\
\hline 8 & No & $\begin{array}{l}\text { Blunt, with } \\
\text { pulse }\end{array}$ & $\begin{array}{l}\text { Abdominal } \\
\text { solid organ } \\
\text { injury }\end{array}$ & None & Yes & $\begin{array}{l}\text { Struck by a } \\
\text { train. No clear } \\
\text { source of } \\
\text { hemorrhage } \\
\text { found that } \\
\text { would have } \\
\text { caused death. }\end{array}$ \\
\hline 9 & Yes & $\begin{array}{l}\text { Blunt, } \\
\text { pulseless }\end{array}$ & Pelvic fracture & None & Yes & $\begin{array}{l}\text { Run over by a } \\
\text { dump truck. } \\
\text { Arrested and } \\
\text { died in the ED. } \\
\text { Difficulty } \\
\text { obtaining } \\
\text { femoral } \\
\text { access for } \\
\text { arterial line } \\
\text { during } \\
\text { resuscitation } \\
\text { likely } \\
\text { secondary to } \\
\text { crush injury to } \\
\text { pelvis. }\end{array}$ \\
\hline
\end{tabular}




\begin{tabular}{|c|c|c|c|c|c|c|}
\hline $\begin{array}{l}\text { Candidate } \\
\#\end{array}$ & $\begin{array}{l}\text { Likely } \\
\text { Candidate }\end{array}$ & $\begin{array}{l}\text { Inclusion } \\
\text { Criteria Met }\end{array}$ & $\begin{array}{l}\text { Primary } \\
\text { Source of } \\
\text { Hemorrhage }\end{array}$ & $\begin{array}{l}\text { Immediate } \\
\text { Surgical } \\
\text { Intervention }\end{array}$ & $\begin{array}{l}\text { Death in } \\
\text { Hospital }\end{array}$ & $\begin{array}{l}\text { Case } \\
\text { Description }\end{array}$ \\
\hline 10 & Yes & $\begin{array}{l}\text { Penetrating, } \\
\text { with pulse }\end{array}$ & $\begin{array}{l}\text { Abdominal } \\
\text { solid organ } \\
\text { injury }\end{array}$ & OR & No & $\begin{array}{l}\text { Stabbed to } \\
\text { abdomen. } \\
\text { Direct to OR } \\
\text { for trauma } \\
\text { laparotomy. } \\
\text { Received } 14 \\
\text { units of blood } \\
\text { product in } 24 \\
\text { hours. }\end{array}$ \\
\hline 11 & No & $\begin{array}{l}\text { Blunt, with } \\
\text { pulse }\end{array}$ & Pelvic fracture & None & Yes & $\begin{array}{l}\text { Run over by } \\
\text { heavy } \\
\text { machinery. } \\
\text { Likely died } \\
\text { from } \\
\text { respiratory } \\
\text { arrest } \\
\text { secondary to a } \\
\text { high spinal } \\
\text { cord injury. }\end{array}$ \\
\hline \multirow[t]{2}{*}{12} & \multirow[t]{2}{*}{ Yes } & \multirow{2}{*}{$\begin{array}{l}\text { Blunt, with } \\
\text { pulse }\end{array}$} & Pelvic fracture & \multirow[t]{2}{*}{ OR } & \multirow[t]{2}{*}{ No } & \multirow{2}{*}{$\begin{array}{l}\text { Pedestrian } \\
\text { struck by a } \\
\text { vehicle. Direct } \\
\text { to OR. } \\
\text { Received } 17 \\
\text { units of blood } \\
\text { product in } 24 \\
\text { hours. }\end{array}$} \\
\hline & & & $\begin{array}{l}\text { Abdominal } \\
\text { solid organ } \\
\text { injury }\end{array}$ & & & \\
\hline \multirow[t]{2}{*}{13} & \multirow[t]{2}{*}{ Yes } & \multirow{2}{*}{$\begin{array}{l}\text { Blunt, with } \\
\text { pulse }\end{array}$} & Pelvic fracture & \multirow[t]{2}{*}{ IR } & \multirow[t]{2}{*}{ No } & \multirow[b]{2}{*}{$\begin{array}{l}\text { MVC. IR } \\
\text { embolization } \\
\text { of right } \\
\text { iliolumbar / } \\
\text { lumbar arteries } \\
\text { successful. } \\
\text { Received } 17 \\
\text { units of blood } \\
\text { product in } 24 \\
\text { hours. }\end{array}$} \\
\hline & & & $\begin{array}{l}\text { Abdominal } \\
\text { solid organ } \\
\text { injury }\end{array}$ & & & \\
\hline \multirow[t]{2}{*}{14} & \multirow[t]{2}{*}{ Yes } & \multirow[t]{2}{*}{$\begin{array}{l}\text { Penetrating, } \\
\text { with pulse }\end{array}$} & $\begin{array}{l}\text { Abdominal } \\
\text { solid organ } \\
\text { injury }\end{array}$ & \multirow[t]{2}{*}{ OR } & \multirow[t]{2}{*}{ No } & \multirow{2}{*}{$\begin{array}{l}\text { Gunshot } \\
\text { wound to } \\
\text { abdomen. } \\
\text { Direct to OR } \\
\text { for trauma } \\
\text { laparotomy. } \\
\text { Received } 33 \\
\text { units of blood } \\
\text { product in } 24 \\
\text { hours. }\end{array}$} \\
\hline & & & $\begin{array}{l}\text { Major } \\
\text { vascular } \\
\text { injury }\end{array}$ & & & \\
\hline
\end{tabular}




\begin{tabular}{|c|c|c|c|c|c|c|}
\hline $\begin{array}{l}\text { Candidate } \\
\#\end{array}$ & $\begin{array}{l}\text { Likely } \\
\text { Candidate }\end{array}$ & $\begin{array}{l}\text { Inclusion } \\
\text { Criteria Met }\end{array}$ & $\begin{array}{l}\text { Primary } \\
\text { Source of } \\
\text { Hemorrhage }\end{array}$ & $\begin{array}{l}\text { Immediate } \\
\text { Surgical } \\
\text { Intervention }\end{array}$ & $\begin{array}{l}\text { Death in } \\
\text { Hospital }\end{array}$ & $\begin{array}{l}\text { Case } \\
\text { Description }\end{array}$ \\
\hline 15 & Yes & $\begin{array}{l}\text { Blunt, with } \\
\text { pulse }\end{array}$ & $\begin{array}{l}\text { Abdominal } \\
\text { solid organ } \\
\text { injury }\end{array}$ & IR & No & $\begin{array}{l}\text { Fall from a } \\
\text { second story } \\
\text { building. } \\
\text { Underwent IR } \\
\text { embolization } \\
\text { for control of a } \\
\text { liver laceration. } \\
\text { Received } 29 \\
\text { units of blood } \\
\text { product in } 24 \\
\text { hours. }\end{array}$ \\
\hline 16 & No & $\begin{array}{l}\text { Blunt, with } \\
\text { pulse }\end{array}$ & $\begin{array}{l}\text { Pelvic fracture } \\
\text { Other - } \\
\text { retroperitoneal } \\
\text { hematoma }\end{array}$ & None & No & $\begin{array}{l}\text { Pedestrian } \\
\text { struck by a } \\
\text { vehicle. } \\
\text { Hypotension } \\
\text { likely } \\
\text { exacerbated by } \\
\text { sedation. No } \\
\text { intervention } \\
\text { required for } \\
\text { hemorrhage. }\end{array}$ \\
\hline 17 & Yes & $\begin{array}{l}\text { Blunt, with } \\
\text { pulse }\end{array}$ & $\begin{array}{l}\text { Abdominal } \\
\text { solid organ } \\
\text { injury } \\
\text { Major } \\
\text { vascular } \\
\text { injury }\end{array}$ & OR & Yes & $\begin{array}{l}\text { MVC. Arrested } \\
\text { in the ED. } \\
\text { Direct to OR. } \\
\text { Received an } \\
\text { OR } \\
\text { resuscitative } \\
\text { thoracotomy } \\
\text { and cross } \\
\text { clamping of } \\
\text { the aorta for } \\
\text { attempted } \\
\text { control of } \\
\text { hemorrhage. } \\
\text { Died in the OR. }\end{array}$ \\
\hline 18 & No & $\begin{array}{l}\text { Blunt, with } \\
\text { pulse }\end{array}$ & $\begin{array}{l}\text { Pelvic fracture } \\
\text { Abdominal } \\
\text { solid organ } \\
\text { injury }\end{array}$ & IR & No & $\begin{array}{l}\text { Pedestrian } \\
\text { struck by a } \\
\text { truck. } \\
\text { Transferred } \\
\text { from another } \\
\text { institution. } \\
\text { Responded to } \\
\text { blood product } \\
\text { resuscitation } \\
\text { and stabilized } \\
\text { prior to IR. }\end{array}$ \\
\hline
\end{tabular}




\begin{tabular}{|c|c|c|c|c|c|c|}
\hline $\begin{array}{l}\text { Candidate } \\
\text { \# }\end{array}$ & $\begin{array}{l}\text { Likely } \\
\text { Candidate }\end{array}$ & $\begin{array}{l}\text { Inclusion } \\
\text { Criteria Met }\end{array}$ & $\begin{array}{l}\text { Primary } \\
\text { Source of } \\
\text { Hemorrhage }\end{array}$ & $\begin{array}{l}\text { Immediate } \\
\text { Surgical } \\
\text { Intervention }\end{array}$ & $\begin{array}{l}\text { Death in } \\
\text { Hospital }\end{array}$ & $\begin{array}{l}\text { Case } \\
\text { Description }\end{array}$ \\
\hline 19 & No & $\begin{array}{l}\text { Blunt, with } \\
\text { pulse }\end{array}$ & $\begin{array}{l}\text { Other - groin } \\
\text { hematoma }\end{array}$ & None & No & $\begin{array}{l}\text { Truck ran over } \\
\text { legs of patient. } \\
\text { Inguinal } \\
\text { laceration was } \\
\text { initially missed } \\
\text { but was } \\
\text { amenable to } \\
\text { external } \\
\text { compression. }\end{array}$ \\
\hline 20 & Yes & $\begin{array}{l}\text { Blunt, with } \\
\text { pulse }\end{array}$ & $\begin{array}{l}\text { Abdominal } \\
\text { solid organ } \\
\text { injury }\end{array}$ & OR & No & $\begin{array}{l}\text { MVC. } \\
\text { Sustained a } \\
\text { Grade } 5 \\
\text { splenic injury } \\
\text { that was taken } \\
\text { to the OR for } \\
\text { definitive } \\
\text { control. } \\
\text { Received } 57 \\
\text { units of blood } \\
\text { products in } 24 \\
\text { hours. }\end{array}$ \\
\hline 21 & Yes & $\begin{array}{l}\text { Blunt, with } \\
\text { pulse }\end{array}$ & $\begin{array}{l}\text { Abdominal } \\
\text { solid organ } \\
\text { injury } \\
\text { Major } \\
\text { vascular } \\
\text { injury }\end{array}$ & OR & No & $\begin{array}{l}\text { Motorbike } \\
\text { crash. Direct to } \\
\text { OR. Received } \\
163 \text { units of } \\
\text { blood product } \\
\text { in } 24 \text { hours. }\end{array}$ \\
\hline
\end{tabular}

Nine of the Likely Candidates were female (64.3\%) and the mean age was 46.1 (STD 18.9). The median ISS for the Likely Candidate group was 31.5 (IQR 26.8). The mean systolic blood pressure on arrival in the ED was $112.7 \mathrm{mmHg}$ (STD $26.4 \mathrm{mmHg}$ ). The mean number of units of blood products within the first 1 hour of arrival in the ED was 5.5 (STD 2.3), and the total within the first 24 hours was 39.2 (STD 40.3) (Table 3). Two of the Likely Candidates received CPR (14.3\%), one received resuscitative thoracotomy with aortic cross clamping (7.1\%), four underwent angiography (28.6\%), and four underwent laparotomy (28.6\%). Eight patients immediately went to either the OR or IR suite from the ED (57.1\%). In total, three of the Likely Candidates died from their injuries (21.4\%) (Table 4). 
Table 3

Clinical variables of Potential and Likely REBOA Candidates

\begin{tabular}{|c|c|c|}
\hline & Potential Candidates (\%) & Likely Candidates (\%) \\
\hline Candidates & $21(3.3)$ & $14(2.2)$ \\
\hline \multicolumn{3}{|l|}{ Sex } \\
\hline Male & $12(57.1)$ & $5(35.7)$ \\
\hline Female & $9(42.9)$ & $9(64.3)$ \\
\hline Mean Age (STD) & $48.8(17.8)$ & $46.1(18.9)$ \\
\hline \multicolumn{3}{|l|}{ Inclusion Criteria } \\
\hline Blunt Trauma with Pulse, SBP $<90$ & $17(80.9)$ & $10(71.4)$ \\
\hline Penetrating Trauma with Pulse, SBP $<90$ & $3(14.3)$ & $3(21.4)$ \\
\hline Blunt Trauma and Pulseless & $1(4.8)$ & $1(7.2)$ \\
\hline Penetrating Trauma and Pulseless & $0(0)$ & $0(0)$ \\
\hline \multicolumn{3}{|l|}{ Mechanism of Trauma } \\
\hline MVC & $3(14.3)$ & $3(21.4)$ \\
\hline MBC & $1(4.8)$ & $1(7.1)$ \\
\hline Pedestrian Struck & $8(38.0)$ & $5(35.8)$ \\
\hline Fall & $2(9.5)$ & $2(14.3)$ \\
\hline Crush & $3(14.3)$ & $0(0)$ \\
\hline ATV/ Dirt Bike & $1(4.8)$ & $0(0)$ \\
\hline GSW & $2(9.5)$ & $2(14.3)$ \\
\hline Stab & $1(4.8)$ & $1(7.1)$ \\
\hline \multicolumn{3}{|l|}{ Main Source of Hemorrhage } \\
\hline Pelvic Fracture & $9(42.9)$ & $6(42.9)$ \\
\hline Abdominal Hemorrhage & $13(61.9)$ & $10(71.4)$ \\
\hline Spleen Injury & 7 (33.3) & $4(28.6)$ \\
\hline Liver Injury & $10(47.6)$ & $9(64.3)$ \\
\hline Kidney Injury & $3(14.3)$ & $2(14.3)$ \\
\hline Lower Extremity Amputation & $0(0)$ & $0(0)$ \\
\hline Major Vascular Injury & $3(14.3)$ & $3(21.4)$ \\
\hline
\end{tabular}




\begin{tabular}{|lll|}
\hline & Potential Candidates (\%) & Likely Candidates (\%) \\
\hline Other & $3(14.3)$ & $0(0)$ \\
\hline
\end{tabular}

Table 4

Interventions and outcomes of Potential and Likely REBOA Candidates

\begin{tabular}{|lll|}
\hline & Potential Candidates & Likely Candidates \\
\hline Median ISS (IQR) & $34(17.8)$ & $31.5(26.8)$ \\
\hline Mean Vitals (STD) & & \\
\hline EHS SBP & $111.8(25.4)$ & $112.7(26.4)$ \\
\hline EHS HR & $106.1(22.3)$ & $104.2(21.5)$ \\
\hline ED SBP & $104.8(40.2)$ & $108(41.7)$ \\
\hline ED HR & $102.7(21.0)$ & $102.4(22.8)$ \\
\hline Mean Blood Products Received (STD) & & \\
\hline At 1 hour & $7.0(5.0)$ & $5.5(2.3)$ \\
\hline At 2 hours & $7.4(8.2)$ & $9.1(9.2)$ \\
\hline At 4 hours & $3.5(4.6)$ & $3.9(5.4)$ \\
\hline At 24 hours & $24.2(40.8)$ & $26.3(47.6)$ \\
\hline Total & $34.6(35.6)$ & $39.2(40.3)$ \\
\hline
\end{tabular}




\begin{tabular}{|lll|}
\hline & Potential Candidates (\%) & Likely Candidates (\%) \\
\hline Interventions & & \\
\hline CPR & $4(19.0)$ & $1(14.3)$ \\
\hline Resuscitative Thoracotomy & $1(4.8)$ & $1(7.1)$ \\
\hline Aortic Cross Clamping & $1(4.8)$ & $4(28.6)$ \\
\hline Angiography & $6(28.6)$ & $4(28.6)$ \\
\hline Laparotomy & $4(19.0)$ & $12(85.7)$ \\
\hline Outcome & & $2(14.3)$ \\
\hline Survival to ED Discharge & $18(85.7)$ & $11(78.6)$ \\
\hline Death in ED & $3(14.2)$ & $1(7.1)$ \\
\hline Survival to Hospital Discharge & $15(71.4)$ & \\
\hline Death in Hospital (post-ED) & $3(14.2)$ & \\
\hline
\end{tabular}

The main sources of infra-diaphragmatic hemorrhage in the Likely Candidates were secondary to an abdominal source in ten patients (71.4\%) (including four spleen injuries, nine liver injuries, and two kidney injuries), pelvic fracture in six patients (42.9\%), and major vascular injury in three patients $(21.4 \%)$. Ten patients had multiple sources of hemorrhage (71.4\%) (Table 2).

Sixty of the cases were assessed independently by both reviewers to assess for inter-rater reliability. Good reliability was found between reviewers for identification of patient candidacy (kappa $=0.659$ ).

\section{Discussion}

\section{Interpretation of Findings}

This study contributes to the evidence evaluating the population-driven need for acquisition of REBOA at Canadian trauma centres. The majority of studies on the efficacy of REBOA in the management of massive hemorrhage from infra-diaphragmatic injury are from other countries, including the USA and Japan, ${ }^{16,23-27}$ in which REBOA is used more commonly; however, the trauma populations, resources, and patterns of injury are vastly different internationally.

In our study, we identified that 14 patients over a three-year period at our institution would have met criteria for use of REBOA during resuscitation. Those that met criteria represented a severely injured group of patients and constituted $2.2 \%$ of the TTAs and $1.1 \%$ of all trauma consults. This can be extrapolated to indicate that REBOA may be utilized in four to five patients per year at our institution. Our findings are consistent with the results of a recent 2021 publication that conducted a similar needs assessment for REBOA at multiple major trauma centres in Edmonton, Canada ${ }^{35}$. This study also found that $1.1 \%$ of the 
study population met eligibility criteria for deployment of REBOA. Although a seemingly small number, this may be clinically significant given that trauma patients are often young, previously healthy individuals with the physiologic reserve to survive this procedure. Additionally, these patients stand to gain many potential high-quality years of life.

In comparison, our institution performed between three to six resuscitative thoracotomies per year $(1.0 \%$ of all trauma consults) during the study time period. Similar to REBOA, RT is a procedure that is performed rarely and requires the need for subsequent immediate definitive management; however, in the setting of infra-diaphragmatic hemorrhage, RT is substantially more invasive and only attempted in patients that have already progressed to cardiac arrest.

Our data indicate that the most common indication for the use of REBOA in our trauma population was for patients that had sustained blunt trauma. The most common mechanism of injury in the REBOA candidates was being a pedestrian struck by a vehicle. This is consistent with Canadian statistics that show the majority of trauma in our country is secondary to blunt trauma. In comparison, the United States has a much higher rate of penetrating trauma. ${ }^{32-34}$ Of note, the percentage of cases that met candidacy criteria in our study is greater than that found in a 2019 study at a US trauma centre that used similar inclusion criteria. The authors found that $0.6 \%$ of the trauma patients seen in their ED per year may have potentially benefitted from REBOA, but over half $(53 \%)$ of which had sustained a penetrating traumatic injury. 20

The main limitation of this study is that, as a retrospective chart review, identification of cases that meet REBOA candidacy does not necessarily reflect that the intervention would have changed the patient outcome. It is important to note that of the 14 Likely Candidates, only three died from their injuries and 11 survived without REBOA. Furthermore, only eight out of the 14 Likely Candidates went directly to the OR or IR suite for attempted embolization. The other six patients did not end up requiring surgery or embolization. However, this reflects the reality that the physician's decision to place REBOA is made early during patient assessment and sometimes without definitive imaging. As with any intervention, its availability provides an option to the care provider that can be employed on a case-by-cases basis according to the physician's clinical judgement that it will improve the outcome of the patient.

It is also notable that the mean SBP on ED arrival for both the Potential and Likely Candidates was greater than $100 \mathrm{mmHg}$. However, a patient's clinical status cannot be reflected by a single value as this is only representative of a moment in their post-injury course. In contrast to these blood pressures, the mean number of units of blood product transfused in the Likely Candidates was 5.5 in the first hour and 39.2 total in the first 24 hours. This meets criteria for massive transfusion at our institution and is indicative of the critical condition of these patients.

One of the strengths of our methods is how we screened for potential REBOA candidates. Other studies have defined the indication for REBOA using ICD-9 or 10 codes $^{20}$ or ISS ${ }^{35}$; however, TTA criteria are more clinically relevant as they are used in real time by physicians during active management of trauma cases. Although there might not have been a change in the outcome for all of the Candidates identified in our 
study, this is more representative of how decisions about interventions are made by physicians in the moment, without a retrospective lens.

\section{Limitations}

This study has a number of limitations. Firstly, as noted, identification of cases that meet REBOA candidacy does not necessarily reflect that the intervention would have changed the patient outcome. This limitation is inherent to the study design but we opted for this approach as an important first step in the assessment of the possible utility of this tool in our trauma population. Secondly, it is possible that cases were missed for inclusion in our study as we only screened TTAs, but we attempted to minimize missed cases by including missed TTAs. Finally, the generalizability of this study is limited in that it was conducted at a single institution. However, our Level 1 trauma centre serves a large urban catchment area, which likely reflects a similar population at other major Canadian trauma centres.

\section{Conclusion And Future Directions}

In conclusion, our study contributes to the evaluation of the potential value of REBOA in trauma management in the Canadian setting. We found that $1.1 \%$ of our annual Canadian trauma population would meet REBOA candidacy, which is similar to the percentage of RTs we perform yearly. While this is a low-volume procedure, it is one that could be potentially life-saving in the most severely injured patients; however, more prospective research is required to determine if the availability of REBOA improves patient outcomes. Additional future directions are conducting a formal needs assessment for the implementation of REBOA involving a cost-benefit analysis and evaluation of the implications to trauma systems at Canadian institutions.

\section{Declarations}

\section{Ethics Approval and Consent to Participate}

Our study received ethics approval from the Fraser Health Research Ethics Board. Consent was not required from patients to perform a chart review.

\section{Consent for Publication}

Not applicable

\section{Availability of Data and Materials}

The datasets used and analyzed during the current study are available from the corresponding author on reasonable request.

\section{Competing Interests}


The authors declare they have no competing interests.

\section{Funding}

Our study received funding from the Royal Columbian Hospital Foundation Fund.

\section{Author's Contributions}

EP prepared the initial literature review and was the primary author of the manuscript. SP was active in data collection and editing of the manuscript. JH participated in case review for the data analysis. VI participated in study preparation, data extraction, and editing. JT provided the methodological expertise and was active in editing of the manuscript. All authors read and approved the final manuscript.

\section{Acknowledgements}

We would like to thank Dr. Samar Hejazi for her assistance with the statistical analysis of the data.

\section{References}

1. Kauvar DS, Lefering R, Wade CE. Impact of hemorrhage on trauma outcome: an overview of epidemiology, clinical presentations, and therapeutic considerations. J Trauma. 2016;60:3-11.

2. Kauvar DS, Wade CE. The epidemiology and modern management of traumatic hemorrhage: US and international perspectives. Crit Care. 2005;9(Suppl 5):1-9.

3. Martin M, Oh J, Currier H, Tai N, Beekley A, Eckert M, et al. An analysis of in-hospital deaths at a modern combat support hospital. J Trauma. 2009;66:51-60.

4. Eastridge BJ, Mabry RL, Seguin P, Cantrell J, Tops T, Uribe P, et al. Death on the battlefield (20012011): implications for the future of combat casualty care. J Trauma Acute Care Surg. 2012;73:4317.

5. Moore LJ, Brenner M, Kozar RA, Pasley J, Wade CE, Baraniuk MS, et al. Implementation of resuscitative endovascular balloon occlusion of the aorta as an alternative to resuscitative thoracotomy for noncompressible truncal hemorrhage. J Trauma Acute Care Surg. 2015;79:523-30.

6. Bansal V, Fortlage D, Lee JG, Costantini T, Potenza B, Coimbra R. Hemorrhage is more prevalent than brain injury in early trauma deaths: the Golden Six Hours. Eur J Trauma Emerg Surg. 2009;35:26-30.

7. Davis JS, Satahoo SS, Butler FK, Dermer H, Naranjo D, Julien K, et al. An analysis of prehospital deaths: who can we save? J Trauma Acute Care Surg. 2014;77:213-8.

8. Borger van der Burg BLS, van Dongen T, Morrison JJ, Hedeman Joosten PPA, DuBose JJ, Horer TM, et al. A systematic review and meta-analysis of the use of resuscitative endovascular balloon occlusion of the aorta in the management of major exsanguination. Eur J Trauma Emerg Surg. 2018;44:53550 .

9. Morrison JJ, Galgon RE, Jansen JO, Cannon JW, Rasmussen TE, Eliason JL. A systematic review of the use of resuscitative endovascular balloon occlusion of the aorta in the management of 
hemorrhagic shock. J Trauma Acute Care Surg. 2016;80:324-34.

10. Kisat M, Morrison JJ, Hashmi ZG, Efron DT, Rasmussen TE, Haider AH. Epidemiology and outcomes of non-compressible torso hemorrhage. J Surg Res. 2013;184:414-21.

11. Morrison JJ, Stannard A, Rasmussen TE, Jansen JO, Tai NRM, Midwinter MJ. Injury pattern and mortality of noncompressible torso hemorrhage in UK combat casualties. J Trauma Acute Care Surg. 2013;75:263-8.

12. Pust GD, Namias N. Resuscitative thoracotomy. Int J Surg. 2016;33:202-8.

13. Stannard A, Eliason JL, Rasmussen TE. Resuscitative endovascular balloon occlusion of the aorta (REBOA) as an adjunct for hemorrhagic shock. J Trauma. 2011;71:1869-72.

14. Brenner ML, Moore LJ, DuBose JJ, Tyson GH, McNutt MK, Albarado RP, Holcomb JB, et al. A clinical series of resuscitative endovascular balloon occlusion of the aorta for hemorrhage control and resuscitation. J Trauma Acute Care Surg. 2013;75(3):506-11.

15. White JM, Cannon JW, Stannard A, Markov NP, Spencer JR, Rasmussen TE. Endovascular balloon occlusion of the aorta is superior to resuscitative thoracotomy with aortic clamping in a porcine model of hemorrhagic shock. Surgery. 2011;150:400-9.

16. DuBose JJ, Scalea TM, Brenner M, Skiada D, Inaba K, Cannon J, et al. The AAST prospective Aortic Occlusion for Resuscitation in Trauma and Acute Care Surgery (AORTA) registry: data on contemporary utilization and outcomes of aortic occlusion and resuscitative balloon occlusion of the aorta (REBOA). J Trauma Acute Care Surg. 2016;81(3):409-19.

17. Markov NP, Percival TJ, Morrison JJ, Ross JD, Scott DJ, Spencer JR, et al. Physiologic tolerance of descending thoracic aortic balloon occlusion in a swine model of hemorrhagic shock. Surgery. 2013;153:848-56.

18. Ryan G, Swift K, Williamson F, Scriven E, Zheng O, Eley R. Feasibility of REBOA - Resuscitative Endovascular Balloon Occlusion of the Aorta - in trauma-related noncompressible torso hemorrhage at two metropolitan trauma centers. Oschsner J. 2018;18(3):201-3.

19. Hughes $\mathrm{CW}$. Use of an intra-aortic balloon catheter tamponade for controlling intra-abdominal hemorrhage in man. Surgery. 1954;36(1):65-8.

20. Dumas RP, Holena DN, Smith BP, Jafari D, Seamon MJ, Reilly PM, et al. Resuscitative Endovascular Balloon Occlusion of the Aorta: assessing need in an urban trauma center. J Surg Res. 2019;233:4139.

21. Joseph B, Ibraheem K, Haider AA, Kulvatunyou N, Tang A, O'Keeffe T, et al. Identifying potential utility of resuscitative endovascular balloon occlusion of the aorta: an autopsy study. J Trauma Acute Care Surg. 2016;81:128-32.

22. Zeismann MT, Rezende-Neto J, McKendy K, Prabhudesai V, Rizoli S, Petrosoniak A. In the Zone: lessons from the first Canadian emergency department application of resuscitative endovascular balloon occlusion of the aorta (REBOA). CJEM. 2019;21(3):430-4.

23. Inoue J, Shiraishi A, Yoshiyuki A, Haruta K, Matsui H, Otomo Y. Resuscitative endovascular balloon occlusion of the aorta might be dangerous in patients with severe torso trauma: a propensity score 
analysis. J Trauma Acute Care Surg. 2016;80(4):559-67.

24. Norii T, Crandall C, Terasaka Y. Survival of severe blunt trauma patients treated with resuscitative endovascular balloon occlusion of the aorta compared with propensity score-adjusted untreated patients. J Trauma Acute Care Surg. 2015;78(4):721-8.

25. Brenner M, Inaba K, Aiolfi A, DuBose J, Fabian T, Bee T, et al. Resuscitative endovascular balloon occlusion of the aorta and resuscitative thoracotomy in select patients with hemorrhagic shock: early results from the American Association for the Surgery of Trauma's Aortic Occlusion in Resuscitation for Truama and Acute Care Surgery registry. J Am Coll Surg. 2018;226:730-40.

26. Joseph B, Zeeshan M, Sakran JV, Hamidi M, Kulvatunyou N, Khan M, et al. Nationwide analysis of resuscitative endovascular balloon occlusion of the aorta in civilian trauma. JAMA Surg. 2019;154(6):500-8.

27. Yamamoto R, Cestero RF, Suzuki M, Funabiki T, Sasaki J. Resuscitative endovascular balloon occlusion of the aorta (REBOA) is associated with improved survival in severely injured patients: a propensity score matching analysis. Am J Surg. 2019;218(6):1162-8.

28. Brenner M, Bulger EM, Perina DG, Henry S, Kang CS, Rotondo MF, et al. Joint statement from the American College of Surgeons Committee on Trauma (ACS COT) and the American College of Emergency Physicians (ACEP) regarding the clinical use of Resuscitative Endovascular Balloon Occlusion of the Aorta (REBOA). Trauma Surg Acute Care Open. 2018;3(1):e000154.

29. Bulger EM, Perina DG, Qasim Z, Beldowicz B, Brenner M, Guyette F, et al. Clinical use of resuscitative endovascular balloon occlusion of the aorta (REBOA) in civilian trauma systems in the USA, 2019: a joint statement from the American College of Surgeons Committee on Trauam, the American College of Emergency Physicians, the National Association of Emergency Medical Services Physicians and the National Association of Emergency Medical Technicians. Trauma Surg Acute Care Open. 2019;4(1):e000376.

30. Lendrum R, Perkins Z, Chana M, Marsden M, Davenport R, Grier G, et al. Pre-hospital Resuscitative Endovascular Balloon Occlusion of the Aorta (REBOA) for exsanguinating pelvic hemorrhage. Resuscitation. 2019;135:6-13.

31. Mill V, Wellme E, Montan C. Trauma patients eligible for resuscitative endovascular balloon occlusion of the aorta (REBOA), a retrospective cohort study. Eur J Trauma Emerg Surg 2020. Online ahead of print.

32. Grinshteyn E, Hemenway D. Violent death rates: the US compared with other high-income OECD countries, 2010. Am J Med. 2016;129(3):266-73.

33. Smith S, McAlister V, Parry N, Power A, Vogt K. Vascular trauma: does experience in the United States apply to a Canadian centre? Can J Surg. 2019;62(6):499-501.

34. Tien HC, Spencer F, Tremblay LN, Rizoli SB, Brenneman FD. Preventable deaths from hemorrhage at a level 1 Canadian trauma center. J Trauma. 2007;62(1):142-6.

35. Eksteen A, O’Dochartaigh D, Odenbach J, Douma MJ, O'Neill K, Anantha R, et al. A gap analysis of the potential use of resuscitative endovascular balloon occlusion of the aorta (REBOA) in trauma at two 
major Canadian Trauma centers. CJEM. 2021;23:36-44.

36. Li W, Petrosoniak A, Ziesmann M. REBOA in Canada: time to shine, or time's up? CJEM 2021; 23: 3-5.

37. Morrison JJ, Ross JD, Rasmussen TE, Midwinter MJ, Jansen JO. Resuscitative endovascular balloon occlusion of the aorta: a gap analysis of severely injury UK combat casualties. Shock. 2014;41(5):388-93.

38. Cannon J, Morrison J, Lauer C, Grabo D, Polk T, Blackbourne L, et al. Resuscitative endovascular balloon occlusion of the aorta (REBOA) for hemorrhagic shock. Mil Med. 2018;183(Suppl 2):55-9.

39. Cheema F, Garcia C, Rivera AG. and Chao E. CE: The use of resuscitative endovascular balloon occlusion of the aorta in treating hemorrhagic shock from severe trauma. Am J Nurs. 2018;118(10):22-8.

40. Napolitano LM. Resuscitative endovascular balloon occlusion of the aorta: indications, outcomes, and training. Crit Care Clin. 2017;33(1):55-70.

\section{Figures}

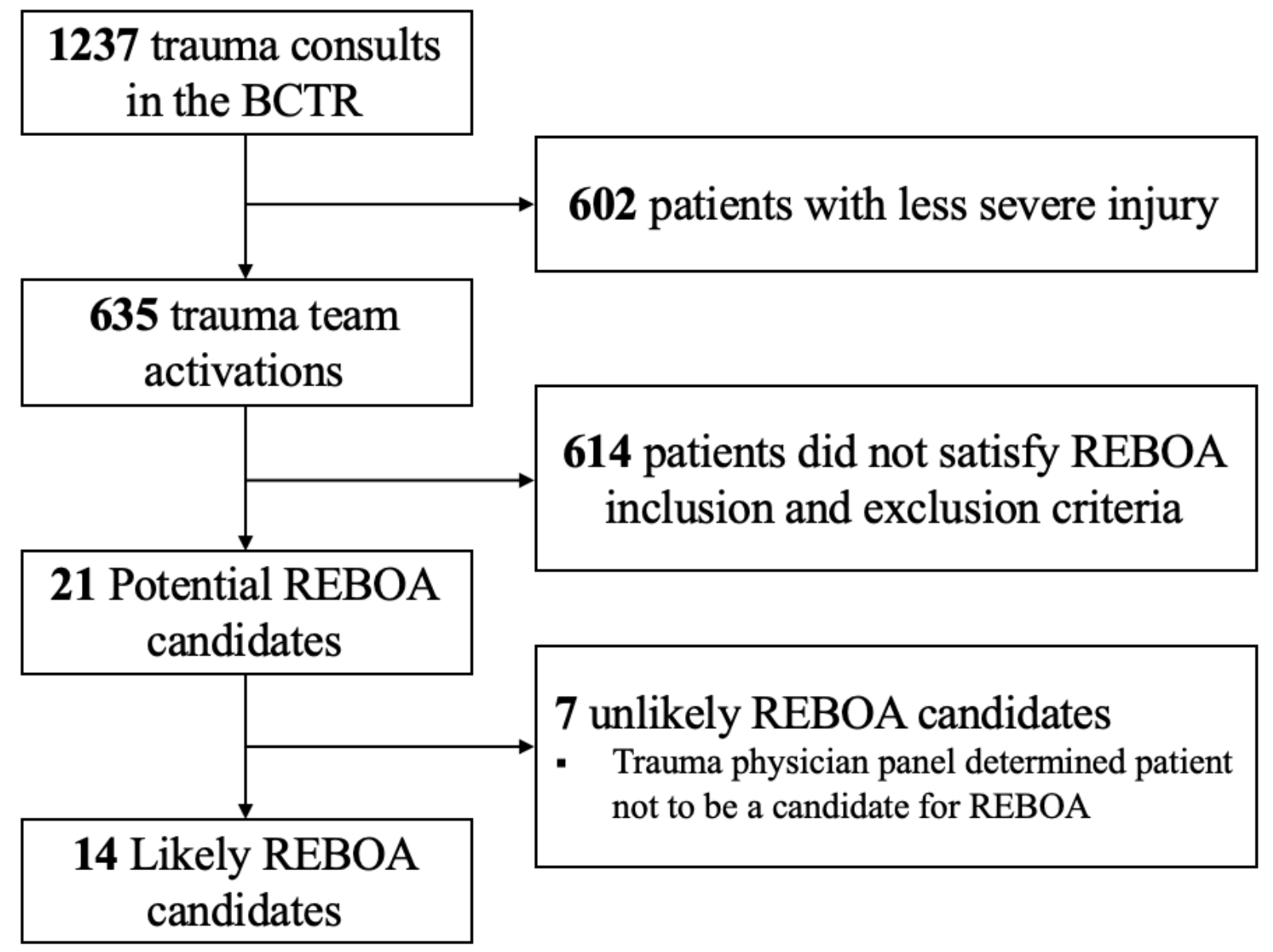

Figure 1 
Flow diagram of assessment for Potential and Likely REBOA candidacy

\section{Supplementary Files}

This is a list of supplementary files associated with this preprint. Click to download.

- AppendixATTACriteria.docx

- AppendixBChartReviewForm.docx 